\title{
Metabolite Profiles in Various Plant Organs of Justicia gendarussa Burm.f. and Its in Vitro Cultures
}

\author{
Putu Indrayoni ${ }^{1}$, Diah Intan Purwanti ${ }^{2}$, Suwidji Wongso ${ }^{2}$, Bambang E.W. Prajogo ${ }^{1}$ and \\ Gunawan Indrayanto ${ }^{1, *}$ \\ 1 Faculty of Pharmacy, Airlangga University, Jl. Dharmawangsa Dalam, Surabaya 60286, Indonesia; \\ indrayoniputu@gmail.com (P.I.); prajogo_ew@yahoo.com (B.E.W.P.) \\ 2 PT. Angler BioChemLab, Plaza Graha Family C-25, Surabaya 60226, Indonesia; intan@anglerlab.net (D.I.P.); \\ dir@anglerlab.net (S.W.) \\ * Correspondence: gunawanindrayanto@yahoo.com; Tel.: +62-81-3305-42324; Fax: +62-31-5025-386
}

Academic Editor: Reinhard Länger

Received: 24 November 2015; Accepted: 4 April 2016; Published: 13 April 2016

\begin{abstract}
Metabolite profiles of plant organs and their in vitro cultures of Justicia gendarussa have been studied by using Ultra Performance Liquid Chromatography-Quadrupole Time-of-Flight-Mass Spectrometry (UPLC-Qtof-MS). Samples of leaves, stems, roots, and shoot cultures showed similar patterns of metabolites, while samples of root cultures gave very different profiles. Concentrations of secondary metabolites in shoot cultures were relatively low compared to those in the leaves and roots of the plants. The results suggested that secondary metabolites in J. gendarussa were biosynthetized in the leaves, then transported to the roots.
\end{abstract}

Keywords: Justicia gendarussa Burm.f.; UPLC-Qtof-MS; plant organs; in vitro cultures; PCA; secondary metabolites accumulation

\section{Introduction}

Justicia gendarussa Burm.f., an evergreen shrub belonging to the family Acanthaceae, is widely distributed in Indonesia, Sri Lanka, India, and Malaysia [1]. This plant has been reported for being cytotoxic [1], immunosuppressive [2], anti-inflammatory, analgesic [3], antioxidant, hepatoprotective [4,5], anti-anxiety [6], antibactericidal [7,8], anti-angiogenic [9], antifungal [10], antisickling [11], anthelmintic [12], larvicidal, adulticidal [13], and for inhibiting both HIV type 1 reverse transcriptase [14] and protein denaturation [15]. n-Butanol fraction of J. gendarussa leaves showed antifertility activity during in vitro and in vivo experiments; the main mechanism was through competitive and reversible inhibition of the spermatozoa hyaluronidase enzyme [16]. Pre-clinical and clinical trials have been conducted on the leaf extract of J. gendarussa in its development as an herbal drug [17-21].

Friedelin, $\beta$-sitosterol, lupeol, $O$-disubstituted aromatic amines, flavonoids, alkaloids, saponins, and phenolic compounds were previously identified in J. gendarussa $[3,7,9,22,23]$. 6,8-di-C- $\alpha$-Larabinosyl-apigenin, 6-C- $\alpha$-L-arabinosyl-8-C- $\beta$-D-xylosyl-apigenin, and justidrusamides A-D were isolated from the leaves of $J$. gendarussa cultivated at Pacet, Indonesia [16,24]. A previous study has shown that metabolite profiles of $J$. gendarussa leaves derived from different locations in Indonesia were affected by their soil nutrients; the concentrations of $\mathrm{Ca}, \mathrm{P}$, and $\mathrm{Cu}$ in the soils could influence its metabolite profiles [25].

The variations in metabolites of different parts of the plant or its in vitro cultures have been reported in the literature for several plant species. Diosgenin was detected in plantlet and shoot cultures of Costus speciosus, but it could not be found in its callus cultures and root cultures [26]. Concentrations of phenolics, flavonoids, alkaloids, and phytosterols in callus cultures of J. gendarussa were equal or slightly increased compared to the original plants [27]. Ma and Gang reported that 
different tissues of turmeric possessed different metabolites profiles [28]. Accumulations of alkaloids in different organs of Lycoris chinensis were different [29]. Variations of secondary metabolites were observed in Juniperus communis [30].

For commercial production of herbal drugs, it is essential to determine where secondary metabolites are accumulated within a plant. The objective of the present study was to investigate the profiles of secondary metabolites in different plant organs of J. gendarussa and its in vitro cultures.

\section{Materials and Methods}

\subsection{Materials and Chemicals}

The J. gendarussa used in this work was of Papua origin and was planted at the campus of Airlangga University, Surabaya, Indonesia. This plant was identified by the Department of Pharmacognosy and Phytochemistry, Faculty of Pharmacy, Airlangga University (voucher no. 22/H3.1.5/DT/2013). Three plants (6 months old) derived from a single plant were cultivated in three different pots (plant 1, 2, and 3), and used as samples. Mature, dark green leaves and stems were collected 4-5 internodes from the terminal bud. Roots of $0-15 \mathrm{~cm}$ length were collected from the main trunk. Murashige Skoog (MS) media supplemented with $0.1 \mathrm{~g} \cdot \mathrm{L}^{-1}$ myo-inositol, $30 \mathrm{~g} \cdot \mathrm{L}^{-1}$ sucrose, and different hormone combinations were used for the in vitro cultures; media A: $6 \mathrm{mg} \cdot \mathrm{L}^{-1}$ benzylaminopurine (BAP), media B: $6 \mathrm{mg} \cdot \mathrm{L}^{-1}$ naphthaleneacetic acid (NAA), and media C: $6 \mathrm{mg} \cdot \mathrm{L}^{-1}$ indolebutyric acid (IBA). Cultures were incubated under continuous light in a growth room illuminated with cool white fluorescent tubes (Philip Lifemax Cool Daylight TLD 36W /54-765) (Philip Lighting, Jakarta, Indonesia) at $25 \pm 2{ }^{\circ} \mathrm{C}$. The subculturing period was 21 days. Plant parts and in vitro cultures were air-dried (Loss on Drying were $1.1 \% \pm 0.3 \%, n=63$ ) and powdered. Table 1 summarizes codes of the samples.

Table 1. Codes of the samples.

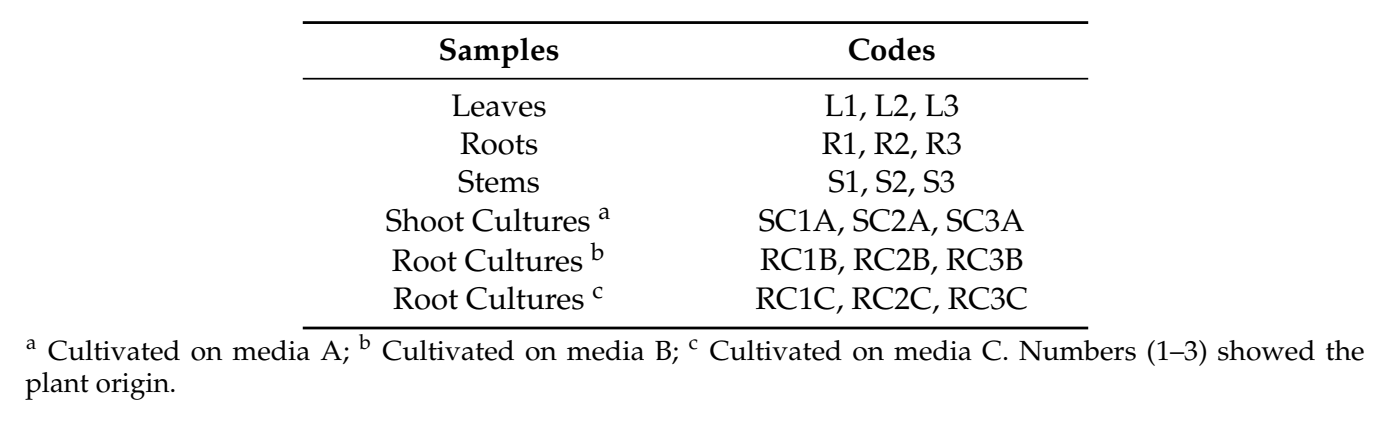

Methanol, ethanol, and formic acid (analytical reagent grade) were from Merck (Darmstadt, Germany). Purified water was from Sigma-Aldrich (St. Louis, MO, USA), acetic acid from J.T. Baker (Phillipsburg, NJ, USA), and $\mathrm{NaOH}$ from Agilent (Agilent solution for HPCE) (Mulgrave, Victoria, Australia). All samples were filtered through the $0.2 \mu \mathrm{m}$ Agilent econo filter polyvinylidene difluoride (PVDF) $13 \mathrm{~mm}$.

\subsection{Preparation of Extracts and Quality Control (QC) Samples}

All the samples of leaves, stems, roots and in vitro cultures of J. gendarussa (Table 1) were extracted in triplicate as described before [25]. The QC samples were prepared according to the published method [31].

\subsection{Instrumentation}

Samples were analyzed using the UPLC Dionex Ultimate 3000 RS LC (Dionex Suftron, GmbH, Thermo Fischer Scientific, Germening, Germany) coupled to the QTOF Bruker Maxis Impact HD 
(Bruker Daltonik, Bremen, Germany), equipped with an Enclosure services interface operating in negative ion mode. It had a mass range of $m / z 50-1000$, the capillary voltage was $2500 \mathrm{~V}$, dry $\mathrm{N}_{2}$ gas flow of $8.0 \mathrm{~L} / \mathrm{min}\left(200^{\circ} \mathrm{C}\right)$, nebulizer pressure 2.0 bars, end plate offset $500 \mathrm{~V}$, collision energy $25 \mathrm{eV}$, and an acquisition time factor of $1 \mathrm{~s}$.

Chromatographic separation was carried out using an Acclaim RSLC 120 C18 column $(2.2 \mu \mathrm{m}$, $120 \AA, 2.1 \times 100 \mathrm{~mm}$ ) (Dionex, Thermo Fischer Scientific, Sunnyvale, CA, USA). The mobile phase consisted of $90 \%$ methanol with $5 \mathrm{mM}$ ammonium acetate and $50 \%$ methanol with $5 \mathrm{mM}$ ammonium acetate. Injection volume was $1.0 \mu \mathrm{L}$. Mass calibration was performed using $1 \mathrm{mM}$ sodium formate/acetate in $50 \%$ isopropanol with $0.2 \%$ formic acid, $\mathrm{HCOO}(\mathrm{NaCOOH})_{1}(\mathrm{~m} / z$ 112.9856), $\mathrm{Ac}(\mathrm{NaAc})_{1}\left(m / z\right.$ 141.0169), and $\mathrm{Ac}(\mathrm{NaF})_{1}(m / z$ 127.0013).

Data analysis and calculations were performed using the following software: Data Analysis 4.1 (SmartFormula, SmartFormula 3D, Isotope Pattern, and Fragmentation Explorer), Profile Analysis 2.1 (PCA and Bucket Statistic Plot), Metabolite Detect 2.0 from Bruker Daltonik, Bremen, Germany, MetFrag (version 2010) [32], Metlin [33], and MassBank [34].

\subsection{Data Processing}

Automatic time alignment was performed on retention time (RT)- $m / z$ pairs of 0.4 to $20 \mathrm{~min}$. Data were grouped automatically into buckets with RT- $m / z$ pairs of $0.5035 \mathrm{~min}$ and $\mathrm{m} / z$ 30.3587; the mass range was 200-700 Da with a mass tolerance 0.05 Da, normalized with the sum of bucket values, pareto-scaled, and a bucket filter of $2 \%$ as described before [25].

The proposed molecular formula was performed using SmartFormula based on the exact mass and isotopic pattern; the proposed fragmentation of the compound was generated using SmartFormula 3D. Then, the fragmentation pattern of the compounds were generated using MetFrag [32] and Fragmentation Explorer.

\subsection{Analytical Method Validation}

Stability testing and method validation (intra-day variability) were performed by injecting sample SC2A at different times: $0 \mathrm{~h}, 12 \mathrm{~h}, 18 \mathrm{~h}$, and $24 \mathrm{~h}$ in triplicate. Principal component analysis (PCA) confirmed that the extracts were stable for at least $24 \mathrm{~h}$, and showed acceptable intra- and inter-day variability.

For checking the reliability of the method for each series of experiments, the QC sample was injected three times at the beginning of the analysis, then regularly every 6-7 samples. Coefficient variations $(\mathrm{CV})$ of the data set were evaluated according to the published method [31]. Our data showed $>85.75 \%$ of the bucket data that showed the CV $<30 \%$. PCA models were cross-validated with full cross-validation and showed no outliers. The tight clustering of the QC samples in the PCA analysis showed the reliability of the method.

\section{Results and Discussion}

PCA analysis of pairs RT and $m / z$ (Figure 1) showed definite discrimination of samples leaves $(\mathrm{L})$, roots (R), stems (S), shoot cultures (SC), and samples of root cultures (RC). Samples L, R, and SC were not well-separated. The total explained variant for the three principle components (PC) were $39.1 \%$. Score plots constructed by using more PCs (up to PC 8) showed similar discrimination patterns (the total explained variants PC 1 to PC 8 was $62.4 \%$ ). 

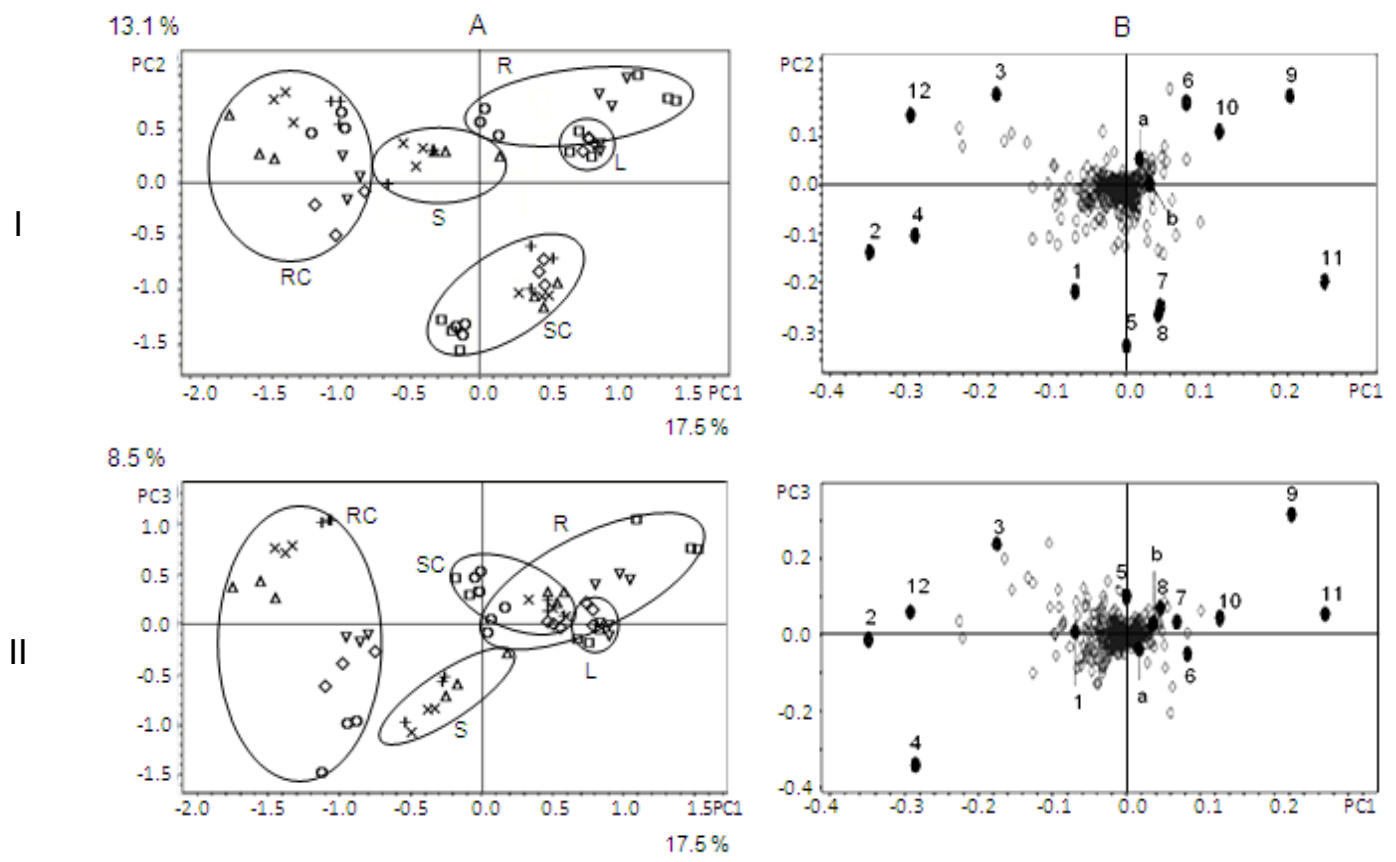

Figure 1. PCA score plots (A) and loading plots (B). Numbers (1-12) and lower case letters (a, b) refer to metabolites as listed in Tables 2 and 3 and Figure 2. RC: root cultures, R: roots, SC: shoot cultures, S: shoots, L: leaves. I: PC1 versus PC2; II: PC1 versus PC3.

The PCA score plot revealed that different combinations of plant growth hormones (media B and C) have relatively no influence on metabolite profiles of the root cultures. PCA score plots showed closeness among the cluster of metabolite profiles of leaves, roots, stems, and shoot cultures, while root cultures produced a very different metabolite profile. These were confirmed by their total ion chromatogram (TIC) patterns (Figure 2). TICs of leaves, roots, stems, and shoot cultures showed almost similar patterns, but root cultures yielded a distinctive profile. Relative intensities of the metabolites as shown by their TICs were confirmed with their bucket statistic plots. TICs also showed that the concentration of metabolites in the shoot cultures was relatively low compared to the leaves and roots of the plants.

The results suggest that secondary metabolites in J. gendarussa are biosynthesized in the leaves and then transported to the stems and roots. 
Samples

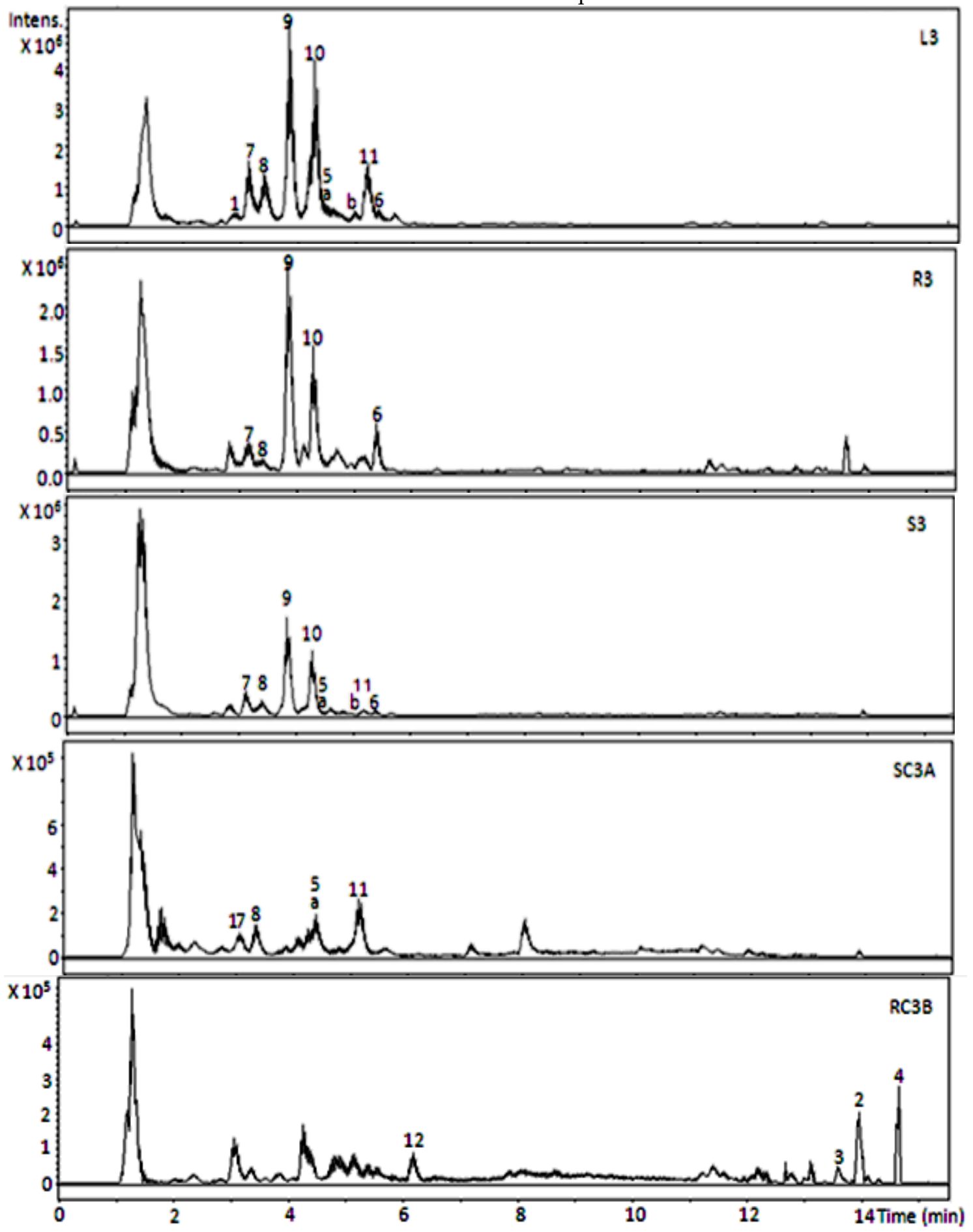

Figure 2. Total ion chromatogram (TIC) of selected samples. Numbers (1-12) and lower case letters $(\mathrm{a}, \mathrm{b})$ refer to metabolites as listed in Tables 2 and 3.

Identification of Metabolites

Loading plots (Figure 1) showed that 12 significant metabolites (1-12) affected the clustering of the samples. Proposed metabolites and their fragmentation patterns are shown in Tables 2 and 3 and Figure 3. 
Metabolites $\mathbf{9}$ and $\mathbf{1 0}$ gave the highest intensity in leaves, roots, and stem samples. The presence of metabolites $\mathbf{a}$ and $\mathbf{b}$ in leaves have previously been reported $[16,24,25]$. Metabolites 3 and 4 were proposed as fatty acids; stearic acid and 9,12-octadecadienoic acid $(Z, Z)$ have been reported to come from the methanolic extract of $J$. wynaadensis analyzed by gas chromatography-mass spectrometry (GC-MS) [35]. Metabolite 6 was proposed as protoberberine alkaloid; different protoberberine alkaloids were previously isolated from aerial parts of Gendarussa vulgaris Nees (synonym of J. gendarussa) [36].<smiles>CCc1ccc(OCC(=O)NCC(=O)OC)cc1</smiles>

(1)

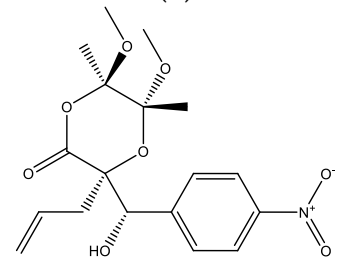

(5)

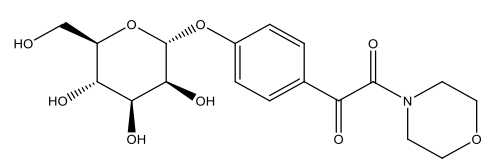

(8)<smiles>CC(C)[C@H](O)C(C(=O)OCCc1ccccc1NC(=O)CCC(=O)O)(C(C)C)C(C)C</smiles>

(a)<smiles>CCCCCCCCCCCCCCC(C)C</smiles>

(3)

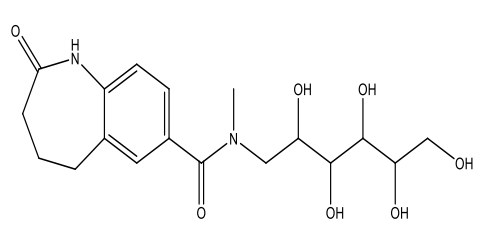

(6)<smiles>C=CC1C2CC3CC(CC)C(C)C(C)C3CC2CC2C(=C)C3CCCC3CC21C</smiles>

(9)

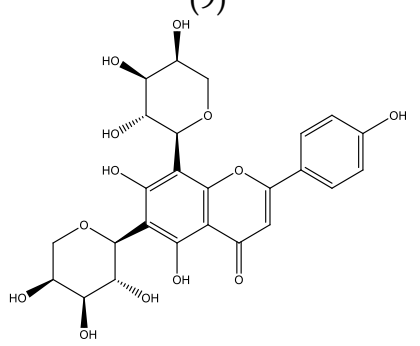

(b)<smiles>CCCCCCCCCCCCCCCC(C)C</smiles>

(4)<smiles>CC(C)C1(C(C)C)C(C)CC1COCc1cc(I)ccc1CC(=O)C1CCC(=O)O1</smiles>

(7)<smiles>CCCCC1CCC(C(C)C(C)C(C)C(C)C(C)C(C)C(C)C(C)CC)CC1</smiles>

(10)

Figure 3. Chemical structures of the proposed metabolites. 
Table 2. Proposed metabolites and their probable elemental formulas.

\begin{tabular}{|c|c|c|c|c|c|}
\hline \multirow[b]{2}{*}{ Metabolites } & \multirow[b]{2}{*}{ RT (min) } & \multirow[b]{2}{*}{ Ions } & Measured $m / z$ & \multirow{2}{*}{$\begin{array}{l}\text { Score (Err[mDa]; } \\
\text { mSigma) Ions a }\end{array}$} & \multirow{2}{*}{$\begin{array}{l}\text { Probable Elemental } \\
\text { Formulas }^{a, b}\end{array}$} \\
\hline & & & $\begin{array}{l}\text { HRMS Ions } \\
(m / z \text { calc. })^{a}\end{array}$ & & \\
\hline 1 & 3.09 & {$[\mathrm{M}-\mathrm{H}]^{-}$} & $\begin{array}{l}251.1039 \\
(251.1037)\end{array}$ & $\begin{array}{c}77 \\
(0.1 ; 2.8)\end{array}$ & $\mathrm{C}_{12} \mathrm{H}_{16} \mathrm{~N}_{2} \mathrm{O}_{4}$ \\
\hline 2 & 13.94 & {$[\mathrm{M}-\mathrm{H}]^{-}$} & $\begin{array}{l}255.2326 \\
(255.2330)\end{array}$ & $\begin{array}{c}88 \\
(-0.3 ; 1.7)\end{array}$ & $\mathrm{C}_{16} \mathrm{H}_{32} \mathrm{O}_{2}$ \\
\hline 3 & 13.64 & {$[\mathrm{M}-\mathrm{H}]^{-}$} & $\begin{array}{l}279.2339 \\
(279.2330)\end{array}$ & $\begin{array}{c}63 \\
(1.0 ; 12.9)\end{array}$ & $\mathrm{C}_{18} \mathrm{H}_{32} \mathrm{O}_{2}$ \\
\hline 4 & 14.66 & {$[\mathrm{M}-\mathrm{H}]^{-}$} & $\begin{array}{l}283.2638 \\
(283.2643)\end{array}$ & $\begin{array}{c}85 \\
(-0.4 ; 0.7)\end{array}$ & $\mathrm{C}_{18} \mathrm{H}_{36} \mathrm{O}_{2}$ \\
\hline 5 & 4.43 & {$[\mathrm{M}-\mathrm{H}]^{-}$} & $\begin{array}{c}380.1339 \\
(380.1351)\end{array}$ & $\begin{array}{c}75 \\
(-1.2 ; 8.7)\end{array}$ & $\mathrm{C}_{18} \mathrm{H}_{23} \mathrm{NO}_{8}$ \\
\hline \multirow{2}{*}{6} & \multirow{2}{*}{5.42} & {$[\mathrm{M}-\mathrm{H}]^{-}$} & $\begin{array}{c}381.1668 \\
(381.1667) \\
\end{array}$ & $\begin{array}{c}100 \\
(-0.1 ; 5.6) \\
\end{array}$ & $\mathrm{C}_{18} \mathrm{H}_{26} \mathrm{~N}_{2} \mathrm{O}_{7}$ \\
\hline & & {$[2 \mathrm{M}-\mathrm{H}]^{-}$} & $\begin{array}{l}763.3405 \\
(763.3407)\end{array}$ & $\begin{array}{c}96 \\
(-0.3 ; 31.8)\end{array}$ & $\mathrm{C}_{36} \mathrm{H}_{52} \mathrm{~N}_{4} \mathrm{O}_{14}$ \\
\hline 7 & 3.09 & {$[\mathrm{M}-\mathrm{H}]^{-}$} & $\begin{array}{c}396.1291 \\
(396.1300)\end{array}$ & $\begin{array}{c}84 \\
(-0.9 ; 7.7)\end{array}$ & $\mathrm{C}_{18} \mathrm{H}_{23} \mathrm{NO}_{9}$ \\
\hline 8 & 3.36 & {$[\mathrm{M}-\mathrm{H}]^{-}$} & $\begin{array}{c}396.1299 \\
(396.1300)\end{array}$ & $\begin{array}{c}100 \\
(0.1 ; 6.4)\end{array}$ & $\mathrm{C}_{18} \mathrm{H}_{23} \mathrm{NO}_{9}$ \\
\hline 9 & 3.84 & {$[\mathrm{M}-\mathrm{H}]^{-}$} & $\begin{array}{c}397.1611 \\
(397.1616)\end{array}$ & $\begin{array}{c}89 \\
(-0.5 ; 2.4)\end{array}$ & $\mathrm{C}_{18} \mathrm{H}_{26} \mathrm{~N}_{2} \mathrm{O}_{8}$ \\
\hline \multirow{2}{*}{10} & \multirow{2}{*}{4.29} & {$[\mathrm{M}-\mathrm{H}]^{-}$} & $\begin{array}{c}397.1614 \\
(397.1616)\end{array}$ & $\begin{array}{c}100 \\
(0.3 ; 1.9)\end{array}$ & $\mathrm{C}_{18} \mathrm{H}_{26} \mathrm{~N}_{2} \mathrm{O}_{8}$ \\
\hline & & {$[2 \mathrm{M}-\mathrm{H}]^{-}$} & $\begin{array}{c}795.3290 \\
(795.3306)\end{array}$ & $\begin{array}{c}48 \\
(1.5 ; 16.3) \\
\end{array}$ & $\mathrm{C}_{36} \mathrm{H}_{52} \mathrm{~N}_{4} \mathrm{O}_{16}$ \\
\hline 11 & 5.18 & {$[\mathrm{M}-\mathrm{H}]^{-}$} & $\begin{array}{l}533.1311 \\
(533.1314)\end{array}$ & $\begin{array}{c}100 \\
(-0.3 ; 6.3)\end{array}$ & $\mathrm{C}_{26} \mathrm{H}_{22} \mathrm{~N}_{4} \mathrm{O}_{9}$ \\
\hline 12 & 6.17 & {$[\mathrm{M}-\mathrm{H}]^{-}$} & $\begin{array}{c}651.2301 \\
(651.2294)\end{array}$ & $\begin{array}{c}100 \\
(0.7 ; 10.0)\end{array}$ & $\mathrm{C}_{31} \mathrm{H}_{40} \mathrm{O}_{15}$ \\
\hline \multirow{2}{*}{$\mathrm{a}$} & \multirow{2}{*}{4.26} & {$[\mathrm{M}-\mathrm{H}]^{-}$} & $\begin{array}{c}368.1357 \\
(368.1351) \\
\end{array}$ & $\begin{array}{c}69 \\
(0.6 ; 5.9) \\
\end{array}$ & $\mathrm{C}_{17} \mathrm{H}_{23} \mathrm{NO}_{8}$ \\
\hline & & {$[2 \mathrm{M}-\mathrm{H}]^{-}$} & $\begin{array}{c}737.2779 \\
(737.2775) \\
\end{array}$ & $\begin{array}{c}100 \\
(0.4 ; 2.1)\end{array}$ & $\mathrm{C}_{34} \mathrm{H}_{46} \mathrm{~N}_{2} \mathrm{O}_{16}$ \\
\hline $\mathrm{b}$ & 5.01 & {$[\mathrm{M}-\mathrm{H}]^{-}$} & $\begin{array}{c}533.1313 \\
(533.1301)\end{array}$ & $\begin{array}{c}67 \\
(-1.3 ; 5.0)\end{array}$ & $\mathrm{C}_{25} \mathrm{H}_{26} \mathrm{O}_{13}$ \\
\hline
\end{tabular}

${ }^{a}$ Data were obtained using Smart Formula 3D; ${ }^{b}$ Elemental formulas were confirmed with their isotope patterns. RT: retention time; HRMS: High Resolution Mass Spectrometry. 
Table 3. Proposed metabolites and their fragmentations.

\begin{tabular}{|c|c|c|c|c|c|c|}
\hline Metabolites & $\begin{array}{l}\text { Score (Chem Spider } \\
\text { a/Pub Chem }{ }^{b} \text { ) }\end{array}$ & $\begin{array}{l}\text { Explained }{ }^{\mathrm{c}} \text { and } \\
\text { MS/MS } \\
\text { Fragment Ions }{ }^{\mathrm{d}}\end{array}$ & 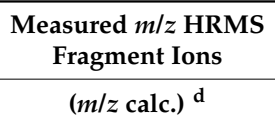 & $\begin{array}{l}\text { Score(Err[mDa]; } \\
\text { mSigma) Fragment } \\
\text { Ions d } \text { d }^{2}\end{array}$ & Proposed Metabolites & $\begin{array}{l}\text { Metabolite IDs and } \\
\text { References }\end{array}$ \\
\hline \multirow{6}{*}{1} & \multirow{6}{*}{$0.991 / 1.0$} & {$\left[\mathrm{C}_{11} \mathrm{H}_{12} \mathrm{NO}_{4}\right]^{-}$} & $\begin{array}{c}222.0778 \\
(222.0772)\end{array}$ & $\begin{array}{c}55 \\
(0.6 ; 21.8)\end{array}$ & \multirow{6}{*}{ Methyl N-\{[4-(aminomethyl)phenoxy]acetyl\} glycinate ${ }^{c}$} & \multirow{6}{*}{$\begin{array}{l}\text { ID } 118625977^{a} \\
\text { CID } 16777361^{b}\end{array}$} \\
\hline & & {$\left[\mathrm{C}_{5} \mathrm{H}_{8} \mathrm{NO}_{4}\right]^{-}$} & $\begin{array}{c}146.0463 \\
(146.0459)\end{array}$ & $\begin{array}{c}75 \\
(0.4 ; 10.8)\end{array}$ & & \\
\hline & & {$\left[\mathrm{C}_{5} \mathrm{H}_{6} \mathrm{NO}_{3}\right]^{-}$} & $\begin{array}{c}128.0355 \\
(128.0353)\end{array}$ & $\begin{array}{c}100 \\
(0.1 ; 1.8)\end{array}$ & & \\
\hline & & {$\left[\mathrm{C}_{7} \mathrm{H}_{8} \mathrm{NO}^{-}\right.$} & $\begin{array}{c}122.0612 \\
(122.0611)\end{array}$ & $\begin{array}{c}66 \\
(0.1 ; 4.3)\end{array}$ & & \\
\hline & & {$\left[\mathrm{C}_{7} \mathrm{H}_{6} \mathrm{NO}^{-}\right.$} & $\begin{array}{c}120.0451 \\
(120.0455)\end{array}$ & $\begin{array}{c}59 \\
(-0.4 ; 3.8)\end{array}$ & & \\
\hline & & {$\left[\mathrm{C}_{3} \mathrm{H}_{5} \mathrm{O}_{2}\right]^{-}$} & $\begin{array}{c}73.0294 \\
(73.0295)\end{array}$ & $\begin{array}{c}63 \\
(-0.1 ; 6.4)\end{array}$ & & \\
\hline 2 & $-/-$ & {$\left[\mathrm{C}_{11} \mathrm{H}_{15} \mathrm{O}\right]^{-}$} & $\begin{array}{c}163.1125 \\
(163.1128)\end{array}$ & $\begin{array}{c}43 \\
(0.3 ; 21.8)\end{array}$ & Unknown $\mathrm{g}$ & \\
\hline \multirow{2}{*}{3} & \multirow{2}{*}{$1.0 / 1.0$} & {$\left[\mathrm{C}_{15} \mathrm{H}_{29} \mathrm{O}_{2}\right]^{-}$} & $\begin{array}{c}241.2177 \\
(241.2173)\end{array}$ & $\begin{array}{c}45 \\
(-0.4 ; 11.1)\end{array}$ & \multirow{2}{*}{ 16-Octadecynoic acid ${ }^{\mathrm{c}, \mathrm{e}}$} & \multirow{2}{*}{$\begin{array}{l}\text { ID } 4472124^{\mathrm{a}} \text {; CID } \\
5312699^{\mathrm{b}} ; \text { ID } 74231 \mathrm{e}\end{array}$} \\
\hline & & {$\left[\mathrm{C}_{14} \mathrm{H}_{27} \mathrm{O}_{2}\right]^{-}$} & $\begin{array}{l}227.2024 \\
(227.2017)\end{array}$ & $\begin{array}{c}24 \\
(0.7 ; 33.5)\end{array}$ & & \\
\hline 4 & $0.911 / 0.911$ & {$\left[\mathrm{C}_{16} \mathrm{H}_{31} \mathrm{O}_{2}\right]^{-}$} & $\begin{array}{c}255.2326 \\
(255.2330)\end{array}$ & $\begin{array}{c}42 \\
(0.4 ; 17.2) \\
\end{array}$ & Stearic acid ${ }^{c, e}$ & $\begin{array}{c}\text { ID 5091 }{ }^{\mathrm{a}} ; \text { CID } \\
164708^{\mathrm{b}} ; \mathrm{ID} 189^{\mathrm{e}} ; \\
\text { C01530 }^{\mathrm{f}}\end{array}$ \\
\hline \multirow{3}{*}{5} & \multirow{3}{*}{$1.0 / 0.835$} & {$\left[\mathrm{C}_{12} \mathrm{H}_{10} \mathrm{NO}_{4}\right]^{-}$} & $\begin{array}{c}232.0611 \\
(232.0615)\end{array}$ & $\begin{array}{c}69 \\
(0.4 ; 13.5)\end{array}$ & \multirow{3}{*}{$\begin{array}{l}\text { (3S,5R,6R)-3-Allyl-3-[(S)-hydroxy(4-nitrophenyl) } \\
\text { methyl]-5,6-dimethoxy-5,6-dimethyl-1,4-dioxan-2-one c }\end{array}$} & \multirow{3}{*}{$\begin{array}{l}\text { ID } 9328344^{\mathrm{a}} \\
{\text { CID } 11153236^{\mathrm{b}}}\end{array}$} \\
\hline & & {$\left[\mathrm{C}_{11} \mathrm{H}_{10} \mathrm{NO}_{3}\right]^{-}$} & $\begin{array}{c}204.0654 \\
(204.0666)\end{array}$ & $\begin{array}{c}26 \\
(1.2 ; 36.7)\end{array}$ & & \\
\hline & & {$\left[\mathrm{C}_{6} \mathrm{H}_{11} \mathrm{O}_{4}\right]^{-}$} & $\begin{array}{c}147.0658 \\
(147.0663)\end{array}$ & $\begin{array}{c}51 \\
(-0.5 ; 6.0)\end{array}$ & & \\
\hline \multirow{2}{*}{6} & \multirow{2}{*}{$0.968 /-$} & {$\left[\mathrm{C}_{6} \mathrm{H}_{11} \mathrm{O}_{5}\right]^{-}$} & $\begin{array}{c}163.0609 \\
(163.0612)\end{array}$ & $\begin{array}{c}64 \\
(0.3 ; 6.2)\end{array}$ & \multirow{2}{*}{$\begin{array}{l}\text { 1-Deoxy-1-\{methyl[(2-oxo-2,3,4,5-tetrahydro-1H-1- } \\
\text { benzazepin-7-yl) carbonyl] amino\}hexitol c }\end{array}$} & \multirow{2}{*}{ ID $34743441^{a}$} \\
\hline & & {$\left[\mathrm{C}_{6} \mathrm{H}_{11} \mathrm{O}_{4}\right]^{-}$} & $\begin{array}{c}147.0663 \\
(147.0663)\end{array}$ & $\begin{array}{c}75 \\
(0.0 ; 4.9)\end{array}$ & & \\
\hline 7 & $1.0 /-$ & {$\left[\mathrm{C}_{6} \mathrm{H}_{11} \mathrm{O}_{5}\right]^{-}$} & $\begin{array}{c}163.0610 \\
(163.0612)\end{array}$ & $\begin{array}{c}89 \\
(0.1 ; 1.5)\end{array}$ & $\begin{array}{l}\text { 1,5-Dideoxy-3-C-\{[(5-hydroxy-2-\{[(5-oxotetra hydro-2- } \\
\text { furanyl) carbonyl] amino\}benzyl) oxy]carbonyl\} pentitol c }\end{array}$ & ID $29814435^{a}$ [25] \\
\hline
\end{tabular}


Table 3. Cont.

\begin{tabular}{|c|c|c|c|c|c|c|}
\hline Metabolites & $\begin{array}{l}\text { Score (Chem Spider } \\
\text { a/Pub Chem }{ }^{\text {b }} \text { ) }\end{array}$ & $\begin{array}{l}\text { Explained }^{\mathrm{c}} \text { and } \\
\text { MS/MS } \\
\text { Fragment Ions } \\
\end{array}$ & $\begin{array}{l}\text { Measured } m / z \text { HRMS } \\
\text { Fragment Ions } \\
(m / z \text { calc. })^{\mathrm{d}}\end{array}$ & $\begin{array}{l}\text { Score(Err[mDa]; } \\
\text { mSigma) Fragment } \\
\text { Ions d }^{~}\end{array}$ & Proposed Metabolites & $\begin{array}{l}\text { Metabolite IDs and } \\
\text { References }\end{array}$ \\
\hline \multirow{2}{*}{8} & \multirow{2}{*}{$0.802 /-$} & {$\left[\mathrm{C}_{6} \mathrm{H}_{11} \mathrm{O}_{5}\right]^{-}$} & $\begin{array}{l}163.0611 \\
(163.0612)\end{array}$ & $\begin{array}{c}85 \\
(-0.1 ; 2.5) \\
\end{array}$ & \multirow{2}{*}{ 4-[4-Morpholinyl(oxo)acetyl] phenyl $\alpha$-D-manno pyranoside ${ }^{c}$} & \multirow{2}{*}{ ID 32768629 a $[25]$} \\
\hline & & {$\left[\mathrm{C}_{4} \mathrm{H}_{5} \mathrm{O}_{3}\right]^{-}$} & $\begin{array}{c}101.0241 \\
(101.0244)\end{array}$ & $\begin{array}{c}50 \\
(0.3 ; 5.0)\end{array}$ & & \\
\hline \multirow{4}{*}{9} & \multirow{4}{*}{$0.841 /-$} & {$\left[\mathrm{C}_{6} \mathrm{H}_{11} \mathrm{O}_{5}\right]^{-}$} & $\begin{array}{c}163.0612 \\
(163.0612)\end{array}$ & $\begin{array}{c}84 \\
(-0.0 ; 5.2)\end{array}$ & \multirow{4}{*}{$\begin{array}{c}(8 \mathrm{~S}, 9 \mathrm{R}, 9 \mathrm{aS}, 10 \mathrm{aR})-5-\text {-Oxo-9-vinyl-1,2,3,8,9,9a,10,10a- } \\
\text { octahydro-5H-imidazo[1,2-a] pyrano [4,3-d] pyridin-8-yl }^{\text {B-D-glucopyranoside }}{ }^{\mathrm{c}}\end{array}$} & \multirow{4}{*}{ ID $26570736^{\mathrm{a}}$} \\
\hline & & {$\left[\mathrm{C}_{5} \mathrm{H}_{9} \mathrm{O}_{5}\right]^{-}$} & $\begin{array}{c}149.0452 \\
(149.0455)\end{array}$ & $\begin{array}{c}77 \\
(0.3 ; 3.5)\end{array}$ & & \\
\hline & & {$\left[\mathrm{C}_{4} \mathrm{H}_{7} \mathrm{O}_{4}\right]^{-}$} & $\begin{array}{c}119.0349 \\
(119.0350)\end{array}$ & $\begin{array}{c}35 \\
(0.1 ; 26.5)\end{array}$ & & \\
\hline & & {$\left[\mathrm{C}_{4} \mathrm{H}_{5} \mathrm{O}_{3}\right]^{-}$} & $\begin{array}{c}101.0246 \\
(101.0244)\end{array}$ & $\begin{array}{c}84 \\
(0.2 ; 1.4)\end{array}$ & & \\
\hline \multirow{3}{*}{10} & \multirow{3}{*}{$0.885 / 0.97$} & {$\left[\mathrm{C}_{6} \mathrm{H}_{11} \mathrm{O}_{5}\right]^{-}$} & $\begin{array}{c}163.0612 \\
(163.0612)\end{array}$ & $\begin{array}{c}86 \\
(-0.0 ; 3.0)\end{array}$ & \multirow{3}{*}{$\begin{array}{c}\text { 2-(\{[(4-Butoxyphenyl)amino](oxo)acetyl\}amino)- } \\
\text { 2-deoxy-D-glucose }{ }^{\mathrm{c}}\end{array}$} & \multirow{3}{*}{$\begin{array}{l}\text { ID } 21249273^{a} \text {; } \\
\text { CID } 24838413^{b}\end{array}$} \\
\hline & & {$\left[\mathrm{C}_{4} \mathrm{H}_{7} \mathrm{O}_{4}\right]^{-}$} & $\begin{array}{c}119.0350 \\
(119.0350) \\
\end{array}$ & $\begin{array}{c}86 \\
(-0.1 ; 2.9) \\
\end{array}$ & & \\
\hline & & {$\left[\mathrm{C}_{4} \mathrm{H}_{5} \mathrm{O}_{3}\right]^{-}$} & $\begin{array}{l}101.0244 \\
(101.0244)\end{array}$ & $\begin{array}{c}52 \\
(0.1 ; 6.4)\end{array}$ & & \\
\hline 11 & $-/-$ & {$\left[\mathrm{C}_{6} \mathrm{H}_{11} \mathrm{O}_{4}\right]^{-}$} & $\begin{array}{c}147.0663 \\
(147.0663)\end{array}$ & $\begin{array}{c}96 \\
(0.0 ; 1.7)\end{array}$ & Unknown $\mathrm{g}$ & \\
\hline 12 & $-1-$ & {$\left[\mathrm{C}_{27} \mathrm{H}_{32} \mathrm{O}_{7}\right]^{-}$} & $\begin{array}{c}468.2158 \\
(468.2154)\end{array}$ & $\begin{array}{c}66 \\
(0.4 ; 20.1)\end{array}$ & Unknown $\mathrm{g}$ & \\
\hline a & $1.0 / 1.0$ & $\begin{array}{c}{\left[\mathrm{C}_{6} \mathrm{H}_{11} \mathrm{O}_{5}\right]^{-}} \\
{\left[\mathrm{C}_{4} \mathrm{H}_{5} \mathrm{O}_{3}\right]^{-}}\end{array}$ & $\begin{array}{c}163,0618 \\
(163,0612) \\
101,0246 \\
(101,0244)\end{array}$ & $\begin{array}{c}64 \\
(0.6 ; 5.9) \\
81 \\
(-0.2 ; 2.5)\end{array}$ & $\begin{array}{c}\text { 3-C-[(\{2-[(3-Carboxypropanoyl)amino]benzyl\}oxy)carbonyl]-1, } \\
\text { 5-dideoxy-L-arabinitol(Justidrusamide A/B })^{\mathrm{C}}\end{array}$ & $\begin{array}{l}\text { ID } 22943323^{\mathrm{a}} ; \text { CID } \\
38352741^{\mathrm{b}} ;[24,25]\end{array}$ \\
\hline \multirow{3}{*}{$\mathbf{b}$} & \multirow{3}{*}{$0.969 / 1.0$} & {$\left[\mathrm{C}_{9} \mathrm{H}_{5} \mathrm{O}_{2}\right]^{-}$} & $\begin{array}{c}145,0301 \\
(145,0295)\end{array}$ & $\begin{array}{c}52 \\
(0.6 ; 24.1)\end{array}$ & \multirow{3}{*}{ 6,8-Di-C-alpha-L-arabino pyranosylapigenin ${ }^{\mathrm{c}, \mathrm{e}}$} & \multirow{3}{*}{$\begin{array}{l}\text { ID 26504074 }{ }^{\mathrm{a}} \\
\text { CID 10918510 } \\
\text { ID } 48669^{\mathrm{e}} ;[16,25]\end{array}$} \\
\hline & & {$\left[\mathrm{C}_{4} \mathrm{H}_{5} \mathrm{O}_{3}\right]^{-}$} & $\begin{array}{c}101.0249 \\
(101.0244)\end{array}$ & $\begin{array}{c}50 \\
(-0.5 ; 8.8)\end{array}$ & & \\
\hline & & {$\left[\mathrm{C}_{3} \mathrm{H}_{5} \mathrm{O}_{3}\right]^{-}$} & $\begin{array}{c}89.0249 \\
(89.0244)\end{array}$ & $\begin{array}{c}52 \\
(-0.5 ; 6.7)\end{array}$ & & \\
\hline
\end{tabular}

Data were obtained using: ${ }^{\text {a MetFrag }(C h e m S p i d e r) ; ~}{ }^{\mathrm{b}}$ MetFrag (PubChem); ${ }^{\mathrm{c}}$ MetFrag; ${ }^{\mathrm{d}}$ Smart Formula 3D; ${ }^{\mathrm{e}}$ Metlin; ${ }^{\mathrm{f}}$ MetFrag (KEGG); ${ }^{\mathrm{g}}$ No match resulted from MetFrag, Metlin, and MassBank. MS/MS: Mass Spectrometry/Mass Spectrometry. 
Metabolite 7 was aminobenzyl alcohol derivate, and 2-aminobenzyl alcohol derivates were previously reported in the leaves of J. gendarussa $[24,25,37]$. Metabolite 8 was identified previously in $J$. gendarussa [25]. Chemical structures of compounds 2, 11, and $\mathbf{1 2}$ could not be matched to any within the database MetFrag [32], Metlin [33], and MassBank [34].

In conclusion, this present work has shown that metabolite profiles in the roots and leaves of $J$. gendarussa are almost identical, but the concentrations of metabolites in shoot cultures seemed very low compared to the leaves. Therefore, it is suggested to use leaves of J. gendarussa as the source for herbal drug raw materials; it seems that the application of tissue cultures as an alternative source for herbal drug production of J. gendarussa is not recommended.

Acknowledgments: We are grateful to Dr. Suciati (Airlangga University) for discussions and English correction.

Author Contributions: All authors have contributed in this work; cultivation of plants and in vitro cultures, sample's processing, extractions were performed at Airlangga University, liquid chromatography-mass spectrometry analysis were performed at PT Angler BioChemLab.

Conflicts of Interest: The authors declare no conflict of interest.

\section{References}

1. Ayob, Z.; Bohari, S.P.; Samad, A.A.; Jamil, S. Cytotoxic Activities against Breast Cancer Cells of Local Justicia gendarussa Crude Extracts. Evid-Based Complement. Altern. Med. 2014. [CrossRef] [PubMed]

2. Arokiyaraj, S.; Perinbam, K.; Agastian, P.; Balaraju, K. Immunosuppresive Effect of Medicinal Plants of Kolli Hills on Mitogen-Stimulated Proliferation of The Human Peripheral Blood Mononuclear Cells in Vitro. Indian J. Pharmacol. 2007, 39, 180-183.

3. Jothimanivannan, C.; Kumar, R.S.; Subramanian, N. Anti-Inflammatory and Analgesic Activities of Ethanol Extract of Aerial Parts of Justicia gendarussa Burm. Int. J. Biol. Chem. 2010, 6, 278-283.

4. Krishna, K.L.; Mruthunjaya, K.; Patel, J.A. Antioxidant and Hepatoprotective Activity of Leaf Extract of Justicia gendarussa Burm. Int. J. Biol. Chem. 2009, 3, 99-110. [CrossRef]

5. Krishna, K.L.; Mruthunjaya, K.; Patel, J.A. Antioxidant and Hepatoprotective Potential of Stem Methanolic Extract of Justicia gendarussa Burm. Int. J. Pharmacol. 2010, 6, 72-80. [CrossRef]

6. Subramanian, N.; Jothimanivannan, C.; Kumar, R.S.; Kameshwaran, S. Evaluation of Anti-anxiety Activity of Justicia gendarussa Burm. Pharmacologia 2013, 4, 404-407.

7. Sivasakthi, A.; Vijayalakshmi, M. An in Vitro Study of Antibactericidal Activity of Some Secondary Metabolites Rich Fraction from The Leaves of Justicia gendarussa. Int. J. Ethnomed. Pharmacol. Res. 2014, 2, $44-50$.

8. Nirmalraj, S.; Ravikumar, M.; Mahendrakumar, M.; Bharath, B.; Perinbam, K. Antibacterial and Anti-Inflammatory Activity of Justicia gendarussa Burm. F. Leaves. J. Plant Sci. 2015, 10, 70-74.

9. Periyanayagam, K.; Umamaheswari, B.; Suseela, L.; Padmini, M.; Ismail, M. Evaluation of Antiangiogenic Effect of the Leaves of Justicia gendarussa (Burm. f) (Acanthaceae) by Chrio Allontoic Membrane Method. Am. J. Infect. Dis. 2009, 5, 180-182. [CrossRef]

10. Sharma, K.K.; Saikia, R.; Kotoky, J.; Kalita, J.C.; Devi, R. Antifungal activity of Solanum melongena L., Lawsoniainermis L. and Justicia gendarussa B. against Dermatophytes. Int. J. PharmTech Res. 2011, 3, 1635-1640.

11. Mpiana, P.T.; Bokota, M.T.; Ndjele, M.B.; Mudogo, V.; Tshibangu, D.S.; Ngbolua, K.N.; Atibu, E.K.; Kwembe, J.T.; Makelele, L.K. Antisickling Activity of Three Species of Justicia from Kisangani (D.R. Congo): J. tenella, J. gendarussa and J. insularis. Int. J. Biol. Chem. Sci. 2010, 4, 1953-1961. [CrossRef]

12. Saha, M.R.; Debnath, P.C.; Rahman, A.; Islam, A.U. Evaluation of in vitro anthelmintic activities of leaf and stem extracts of Justicia gendarussa. Bangladesh J. Pharmacol. 2012, 7, 50-53. [CrossRef]

13. Senthilkumar, N.; Varma, P.; Gurusubramanian, G. Larvicidal and Adulticidal Activities of Some Medicinal Plants against the Malarial Vector Anopheles stephensi (Liston). Parasitol. Res. 2009, 104, 237-244. [CrossRef] [PubMed]

14. Woradulayapinij, W.; Soonthornchareonnon, N.; Wiwat, C. In vitro HIV type 1 reverse transcriptase inhibitory activities of Thai medicinal plants and Canna indica L. rhizomes. J. Ethnopharmacol. 2005, 101, 84-89. [CrossRef] [PubMed] 
15. Patel, S.S.; Zaveri, M.N. Trypsin and Protein Denaturation Inhibitory Activity of Different Fractionation and Isolated Compound of Leaf and Root of Justicia Gendarussa. IJPSR 2014, 5, 5564-5571.

16. Prajogo, B.E. Aktivitas Antifertilitas Flavonoid Daun Justicia gendarussa Burm.f.: Penelitian Eksperimental Pencegahan Penetrasi Spermatozoa Mencit dalam Proses Fertilisasi in Vitro (in Indonesian). Ph.D. Thesis, Airlangga University, Surabaya, Indonesia, 2002.

17. Prajogo, B.E.; Widjiati, T.M. Pengaruh Fraksi Polifenol Gendarussa vulgaris Nees pada Penurunan Aktivitas Hialuronidase Spermatozoa Mencit melalui Uji Fertilitas in Vitro (in Indonesian). Laporan Penelitian Ilmu Pengetahuan Dasar, Lembaga Penelitian Universitas Airlangga, Surabaya, Indonesia, 2002.

18. Prajogo, B.E.; Ifadotunnikmah, F.; Febriyanti, A.P.; Jusak, N. Efek Fase Air Daun Gandarusa (Justicia gendarussa Burm.f.) pada Fungsi Hati dan Fungsi Ginjal Kelinci Jantan (Uji Toksisitas Fase Air Daun Gandarusa sebagai Bahan Kontrasepsi Pria) (in Indonesian). Vet. Med. 2008, 1, 79.

19. Prajogo, B.E.; Juliaan, F.; Hinting, A.; Pramesti, M.P.; Anggraeni, M.; Radjaram, A.; Musta'ina. Laporan Pelaksanaan Uji Klinik Fase I; (in Indonesian). Universitas Airlangga dan Badan Koordinasi Keluarga Berencana Nasional: Surabaya, Indonesia, 2008.

20. Prajogo, B.E.; Juliaan, F.; Hinting, A.; Pramesti, M.P.; Anggraeni, M.; Radjaram, A.; Musta'ina. Laporan Pelaksanaan Uji Klinik Fase II; (in Indonesian). Universitas Airlangga dan Badan Koordinasi Keluarga Berencana Nasional: Surabaya, Indonesia, 2009.

21. Prajogo, B.E.; Juliaan, F.; Hinting, A.; Pramesti, M.P.; Anggraeni, M.; Radjaram, A.; Musta'ina. Laporan Pelaksanaan Uji Klinik Fase III; (in Indonesian). Universitas Airlangga dan Badan Koordinasi Keluarga Berencana Nasional: Surabaya, Indonesia, 2011.

22. Satapathy, A.K.; Gunasekaran, G.; Sahoo, S.C.; Amit, K.; Rodrigues, P.V. Corrosion Inhibition by Justicia gendarussa Plant Extract in Hydrochloric Acid Solution. Corros. Sci. 2009, 51, 2848-2856. [CrossRef]

23. Shikha, P.; Latha, P.G.; Suja, S.R.; Anuja, G.I.; Shyamal, S.; Shine, V.J.; Sini, S.; Kumar, N.M.; Rajasekaran, S. Anti-inflammatory and antinociceptive activity of Justicia gendarussa Burm. f. Leaves. Indian J. Nat. Prod. Resour. 2010, 1, 456-461.

24. Kiren, Y.; Deguchi, J.; Hirasawa, Y.; Morita, H.; Prajogo, B.E. Justidrusamides A-D, New 2-Aminobenzyl Alcohol Derivatives from Justicia gendarussa. J. Nat. Med. 2014, 68, 754-758. [CrossRef] [PubMed]

25. Ningsih, I.Y.; Purwanti, D.I.; Wongso, S.; Prajogo, B.E.; Indrayanto, G. Metabolite Profiling of Justicia gendarussa Burm. f. Leaves Using UPLC-UHR-QTOF-MS. Sci. Pharm. 2015, 83, 489-500. [CrossRef] [PubMed]

26. Indrayanto, G.; Setiawan, B.; Cholies, N. Differential Diosgenin Accumulation in Costus speciosus and its Tissue Cultures. Planta Medica Lett. 1994, 60, 483-484. [CrossRef] [PubMed]

27. Bhagya, N.; Chandrashekar, K.R. In Vitro Production of Bioactive Compounds from Stem and Leaf Explants of Justicia gendarussa Burm. f. Asian J. Pharm. Clin. Res. 2013, 6, 100-105.

28. Ma, X.; Gang, D.R. Metabolic Profiling of Turmeric (Curcuma longa L.) Plants Derived from in Vitro Micropropagation and Conventional Greenhouse Cultivation. J. Agric. Food Chem. 2006, 54, 9573-9583. [CrossRef] [PubMed]

29. Mu, H.M.; Wang, R.; Li, X.D.; Jiang, Y.M.; Peng, F.; Xia, B. Alkaloid accumulation in different parts and ages of Lycoris chinensis. Z. Naturforschung C. 2010, 65, 458-462. [CrossRef]

30. Butkienė, R.; Būdienė, J.; Judžentienè, A. Variation of Secondary Metabolites (Essential Oils) in Various Plant Organs of Juniperus communis L. Wild Growing in Lithuania. Balt. For. 2015, 21, 59-64.

31. Want, E.J.; Wilson, I.D.; Gika, H.; Theodoridis, G.; Plumb, R.S.; Shockcor, J.; Holmes, E.; Nicholson, J.K. Global Metabolic Profiling Procedures for Urine Using UPLC-MS. Nat. Protoc. 2010, 5, 1005-1018. [CrossRef] [PubMed]

32. MetFrag. Available online: http://msbi.ipb-halle.de/MetFrag/ (accessed on 26 August-5 September 2015).

33. Metlin. Available online: https://metlin.scripps.edu/index.php (accessed on 26 August-5 September 2015).

34. MassBank. Available online: http://www.massbank.jp/index.html (accessed on 26 August-5 September 2015).

35. Ponnamma, S.U.; Manjunath, K. GC-MS Analysis of Phytocomponents in The Methanolic Extract of Justicia wynaadensis (Nees) T. Anders. Int. J. Pharm. Bio. Sci. 2012, 3, 570-576. 
36. Lu, S.; Zhang, G. Alkaloids from Gendarussa vulgaris Nees. Nat. Prod. Res. 2008, 22, 1610-1613. [CrossRef] [PubMed]

37. Chakravarty, A.K.; Dastidar, P.P.; Pakrashi, S.C. Simple Aromatic Amines from Justicia gendarussa: 13C NMR Spectra of The Bases and Their Analogues. Tetrahedron 1982, 18, 1797-1802. [CrossRef]

(c) 2016 by the authors; licensee MDPI, Basel, Switzerland. This article is an open access article distributed under the terms and conditions of the Creative Commons Attribution (CC-BY) license (http:/ / creativecommons.org/licenses/by/4.0/). 